Supporting Information

Defect-Rich $\mathrm{MoO}_{3}$ Nanobelt Cathode for High-performance Hybrid

\title{
Alkali/acid $\mathrm{Zn}-\mathrm{MoO}_{3}$ Rechargeable Battery
}

Pingwei Cai ${ }^{a}$, Junxiang Chen ${ }^{a}$, Yichun Dinga, Yangjie Liü, and Zhenhai Wen**

a CAS Key Laboratory of Design and Assembly of Functional Nanostructures, Fujian

Provincial Key Laboratory of Nanomaterials, Fujian Institute of Research on the Structure of

Matter, Chinese Academy of Sciences, Fuzhou 350002, China

Corresponding Authors: Zhenhai Wen

Email: Wen@fjirsm.ac.cn

Number of pages: 25

Number of tables: 1

Number of figures: 22 


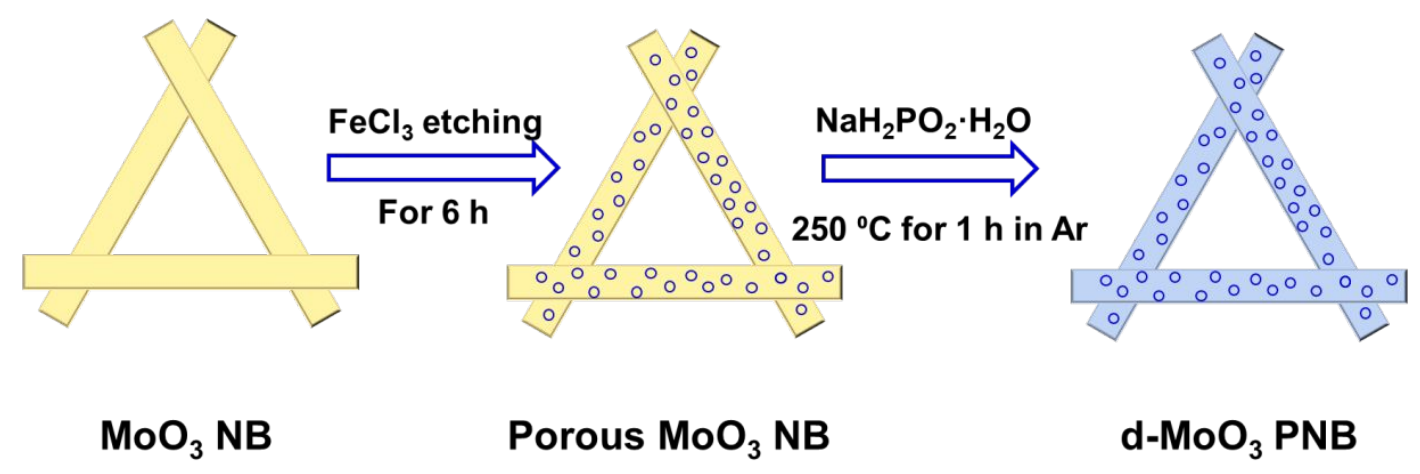

Figure S1 Schematic diagram of the synthesis of d-MoO $\mathrm{MN}_{3} \mathrm{PN}$. 

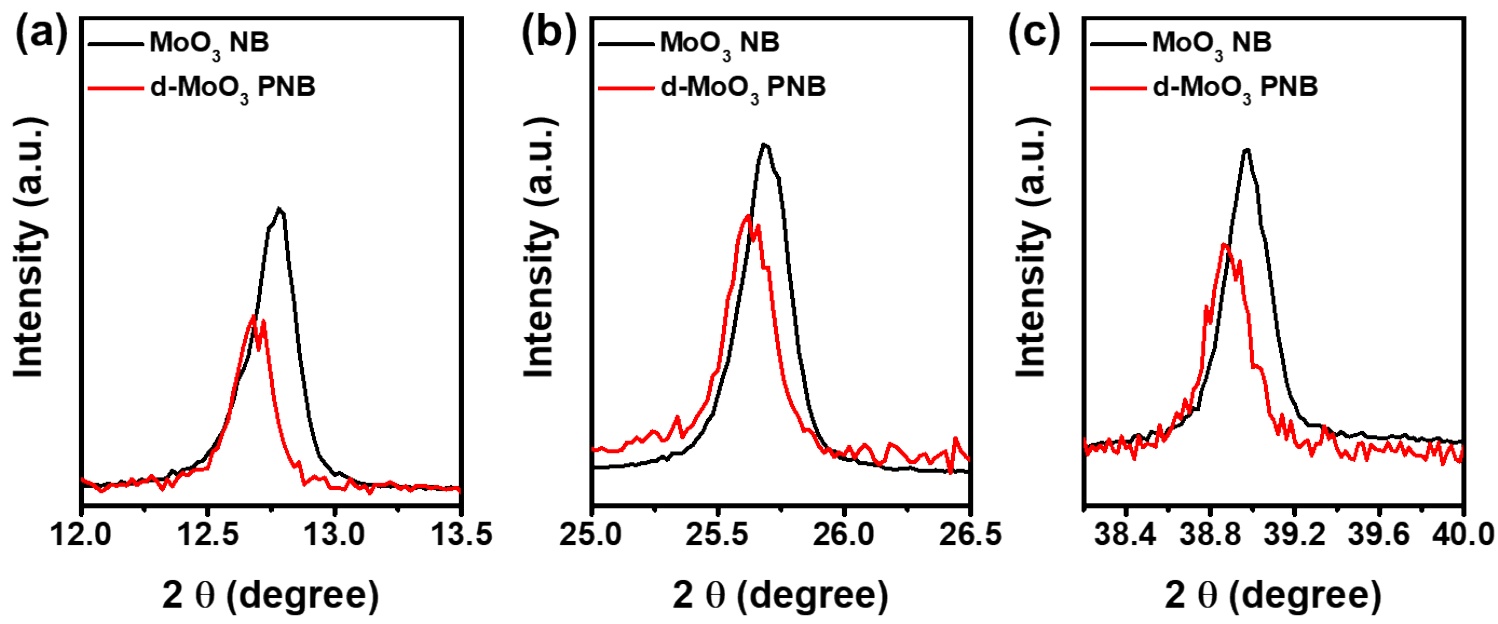

Figure S2 (a-c ) Magnified XRD patterns of $\mathrm{MoO}_{3} \mathrm{NB}$ and d- $\mathrm{MoO}_{3} \mathrm{PNB}$ at different ranges. 

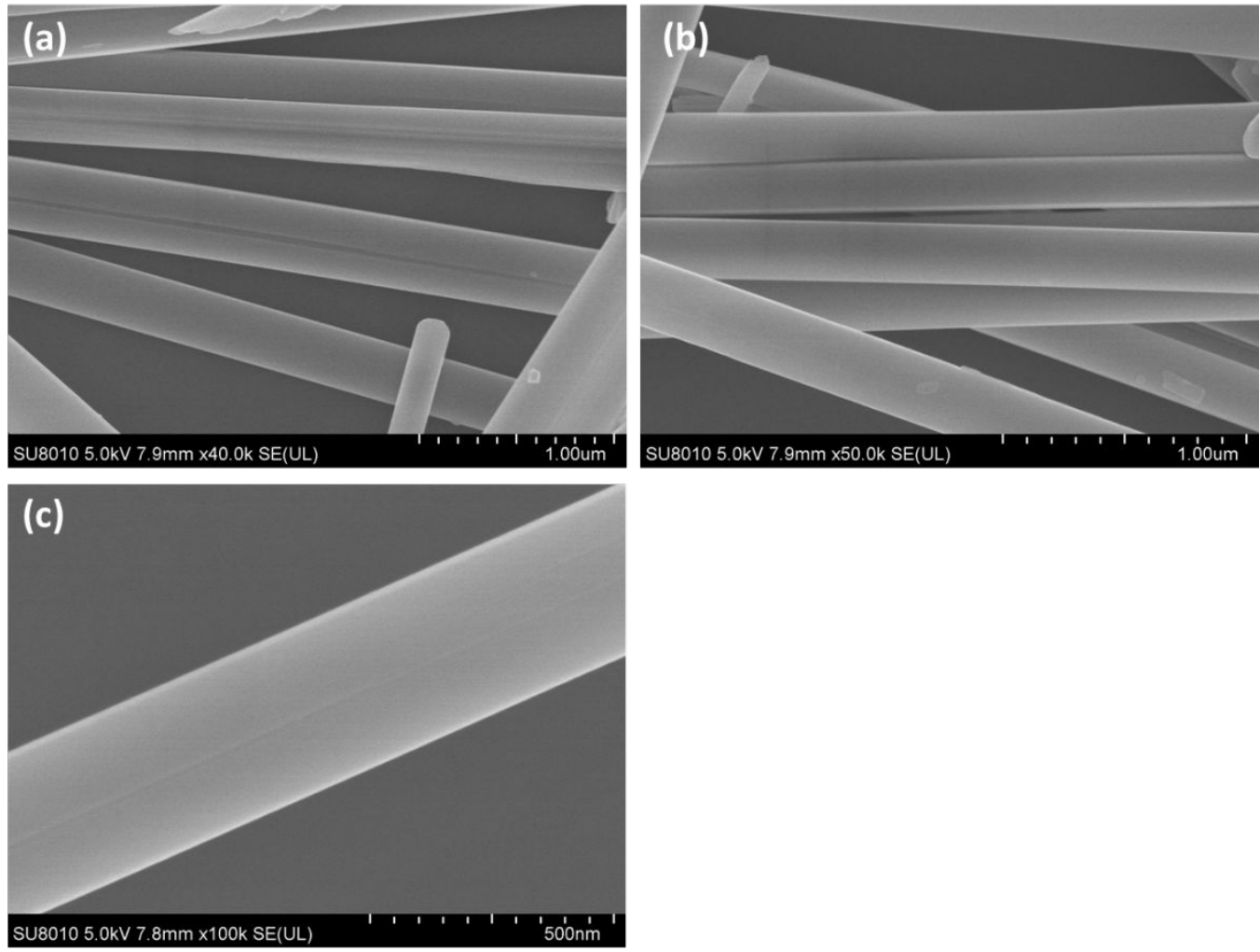

Figure S3 (a-c) SEM images of $\mathrm{MoO}_{3} \mathrm{NB}$ with different magnifications. 

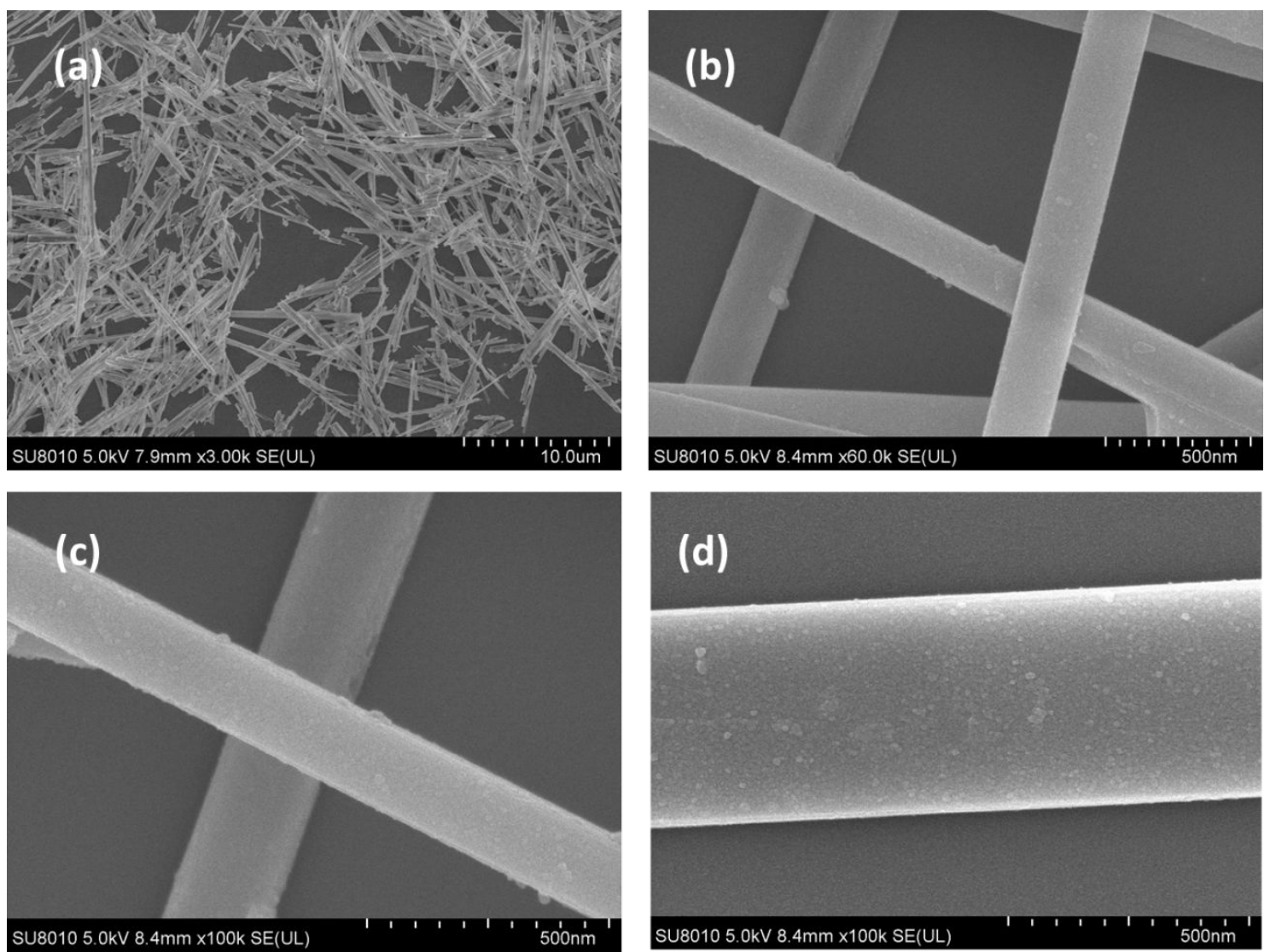

Figure S4 (a-d) SEM images of d- $\mathrm{MoO}_{3}$ PNB with different magnifications. 
(a)

(b)

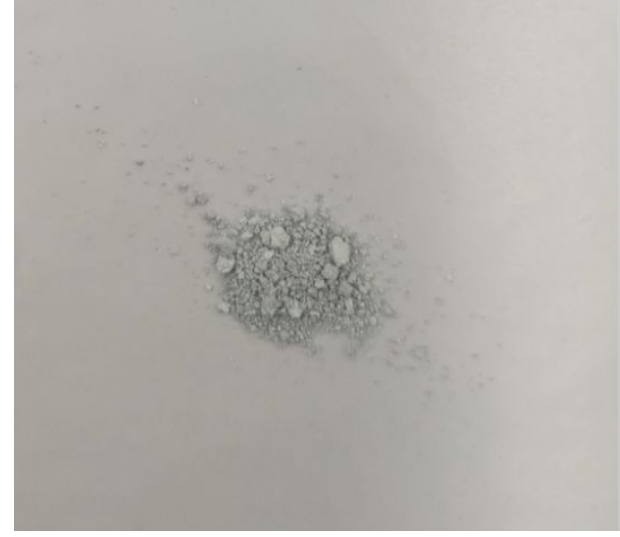

Figure S5 Digital graph of $\mathrm{MoO}_{3} \mathrm{NB}$ (a) and d- $\mathrm{MoO}_{3} \mathrm{PNB}$ (b). 


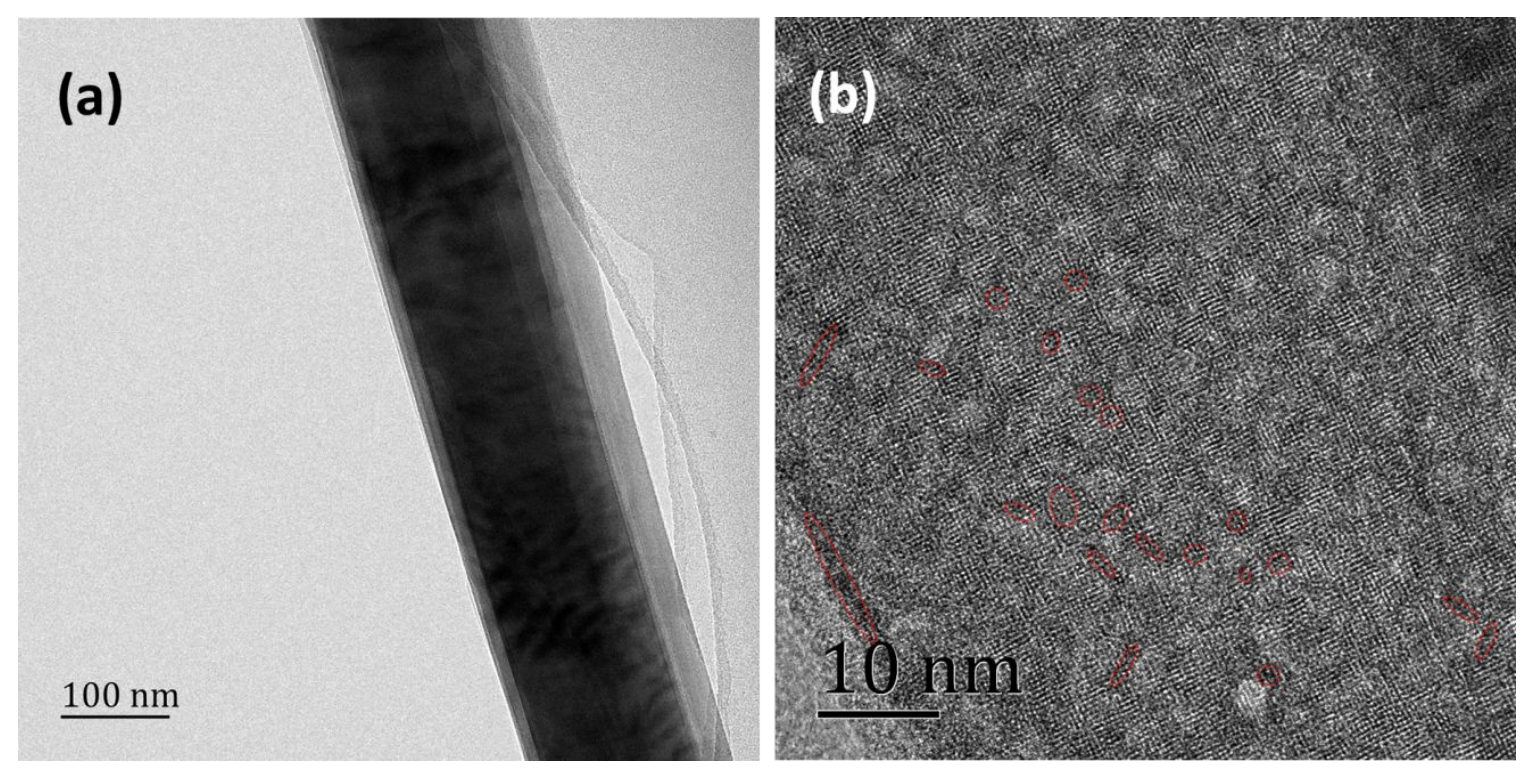

Figure S6 TEM images of d-MoO 3 PNB. (a) Low-resolution image; (b) High-resolution image. 

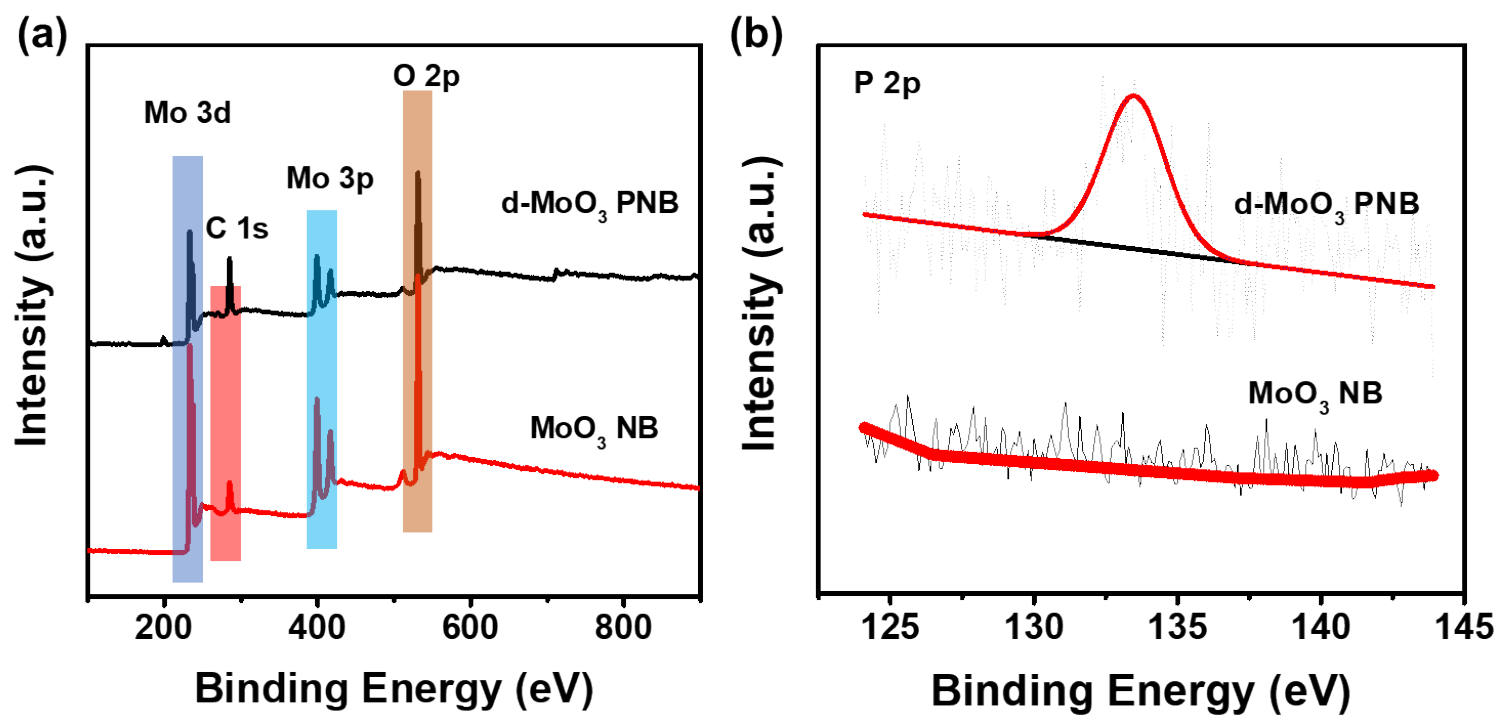

Figure S7 XPS survey spectroscopy (a), and P 2p core level (b) of $\mathrm{MoO}_{3} \mathrm{NB}$ and d- $\mathrm{MoO}_{3}$ PNB. 


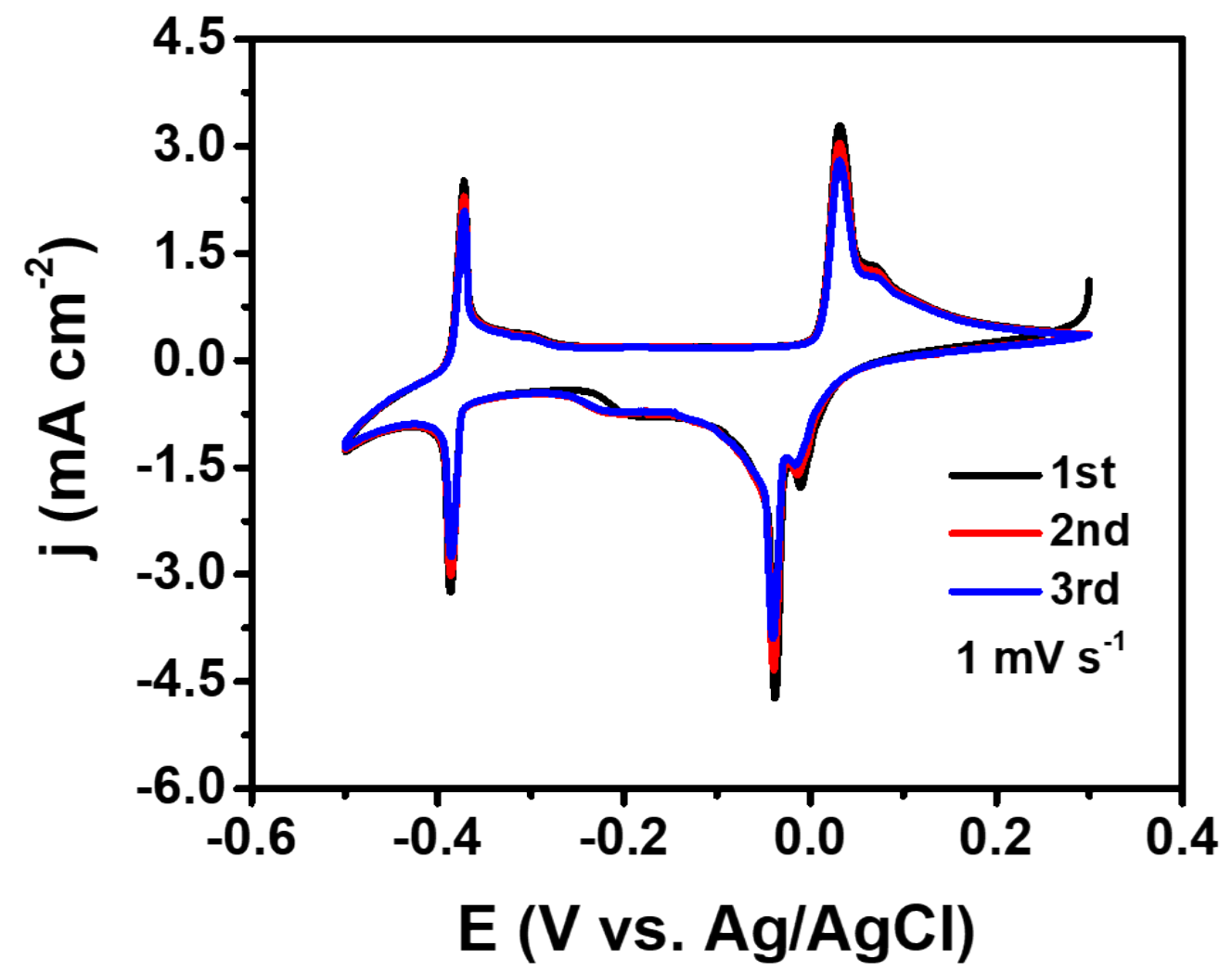

Figure S8 CV curves of the d- $\mathrm{MoO}_{3} \mathrm{PNB}$ electrode for the first three cycles at a scan rate of $1 \mathrm{mV} \mathrm{s}^{-1}$. 

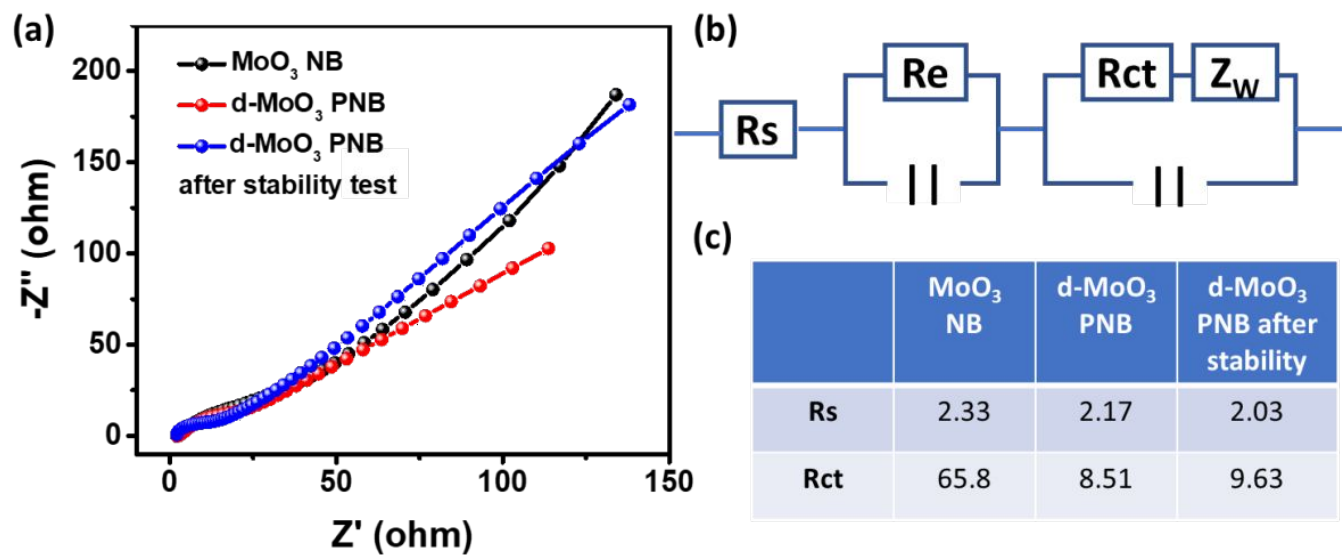

Figure S9 EIS of $\mathrm{MoO}_{3} \mathrm{NB}$ and d- $\mathrm{MoO}_{3}$ PNB recorded at open circuit potential in a threeelectrode system. (a) Nyquist curves; (b) Corresponding fitting circuit; (c) The source resistance $\left(\mathrm{R}_{\mathrm{s}}\right)$ and charge transfer resistance $\left(\mathrm{R}_{\mathrm{ct}}\right)$.

According to the fitting EIS, the source resistance (Rs) and charge transfer resistance (Rct) can be estimated. The $\mathrm{MoO}_{3} \mathrm{NB}(2.33 \mathrm{ohm})$ and $\mathrm{d}-\mathrm{MoO}_{3}(2.17 \mathrm{ohm})$ PNB show a similar Rs value, while the $\mathrm{d}-\mathrm{MoO}_{3} \mathrm{PNB}$ has a lower Rct $(8.51 \mathrm{ohm})$ than that of $\mathrm{MoO}_{3} \mathrm{NB}(65.8 \mathrm{ohm})$, indicating that the $\mathrm{d}-\mathrm{MoO}_{3}$ has a more favorable reactivity than that of $\mathrm{MoO}_{3} \mathrm{NB}$. Moreover, the Rs and Rct of d- $\mathrm{MoO}_{3}$ PNB after the stability test are 2.03 and $9.63 \mathrm{ohm}$, which are very close to the initial values, manifesting the stability of $\mathrm{d}-\mathrm{MoO}_{3}$ PNB for $\mathrm{H}^{+}$ insertion/deinsertion. 
(a)

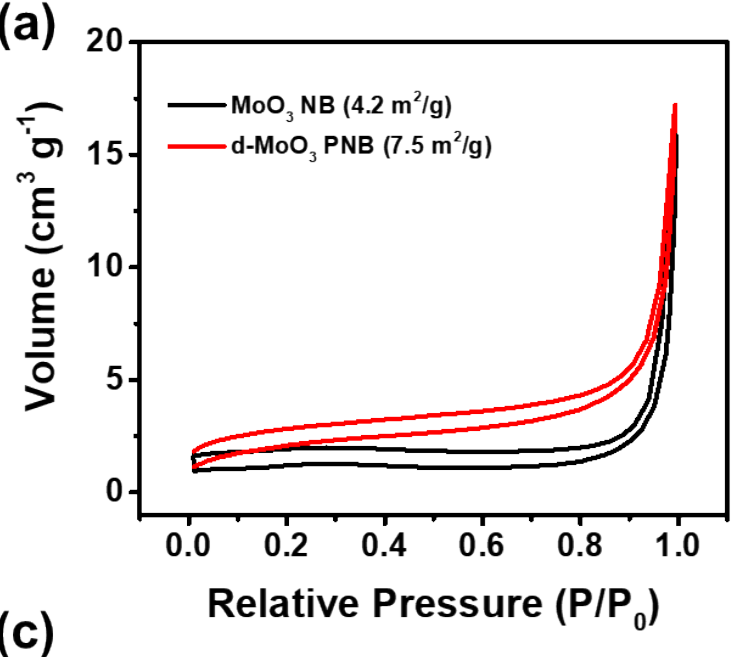

(c)

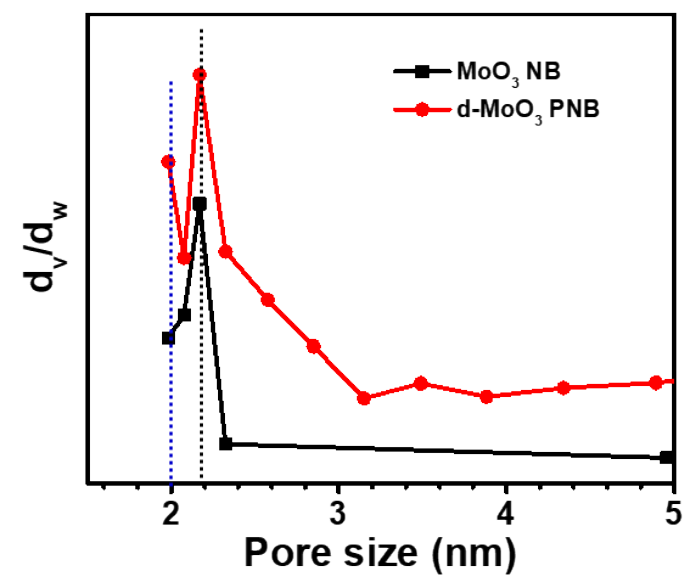

(b)

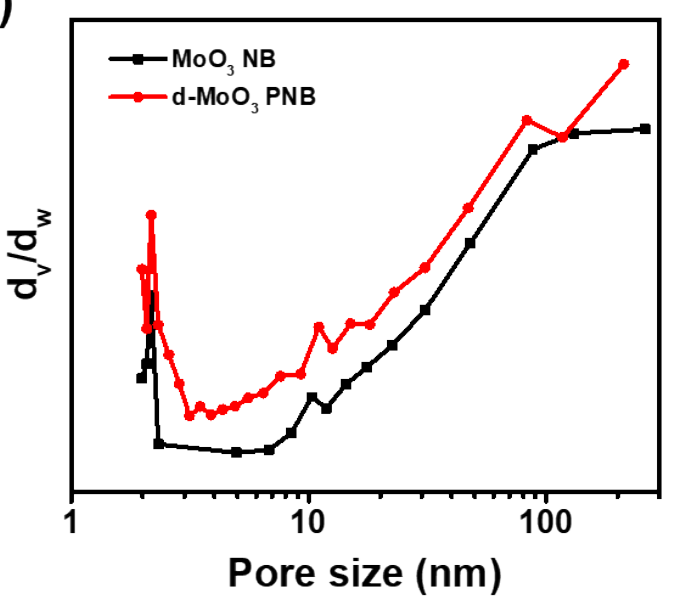

Figure S10 (a) $\mathrm{N}_{2}$ adsorption and desorption curves; (b) Corresponding pore size distribution curves of $\mathrm{MoO}_{3} \mathrm{NB}$ and d-MoO${ }_{3} \mathrm{PNB}$; (c) The magnified Figure of Figure S10b.

The d- $\mathrm{MoO}_{3}$ PNB shows a larger BET surface area $\left(7.5 \mathrm{~m}^{2} \mathrm{~g}^{-1}\right)$ than that of $\mathrm{MoO}_{3} \mathrm{NB}(4.2$ $\mathrm{m}^{2} \mathrm{~g}^{-1}$ ) from the $\mathrm{N}_{2}$ adsorption and desorption curves (Figure S10a). Figure S10b displays the pore size distribution curves of $\mathrm{MoO}_{3} \mathrm{NB}$ and d-MoO 3 PNB, respectively, the pore size of d$\mathrm{MoO}_{3} \mathrm{PNB}$ is centered at about $2.2 \mathrm{~nm}$ and $2 \mathrm{~nm}$ (Figure $\mathrm{S} 10 \mathrm{c}$ ), while $\mathrm{MoO}_{3} \mathrm{NB}$ only shows a peak at about $2.2 \mathrm{~nm}$, indicating that the etching and doping treatment tends to generate micropores. 


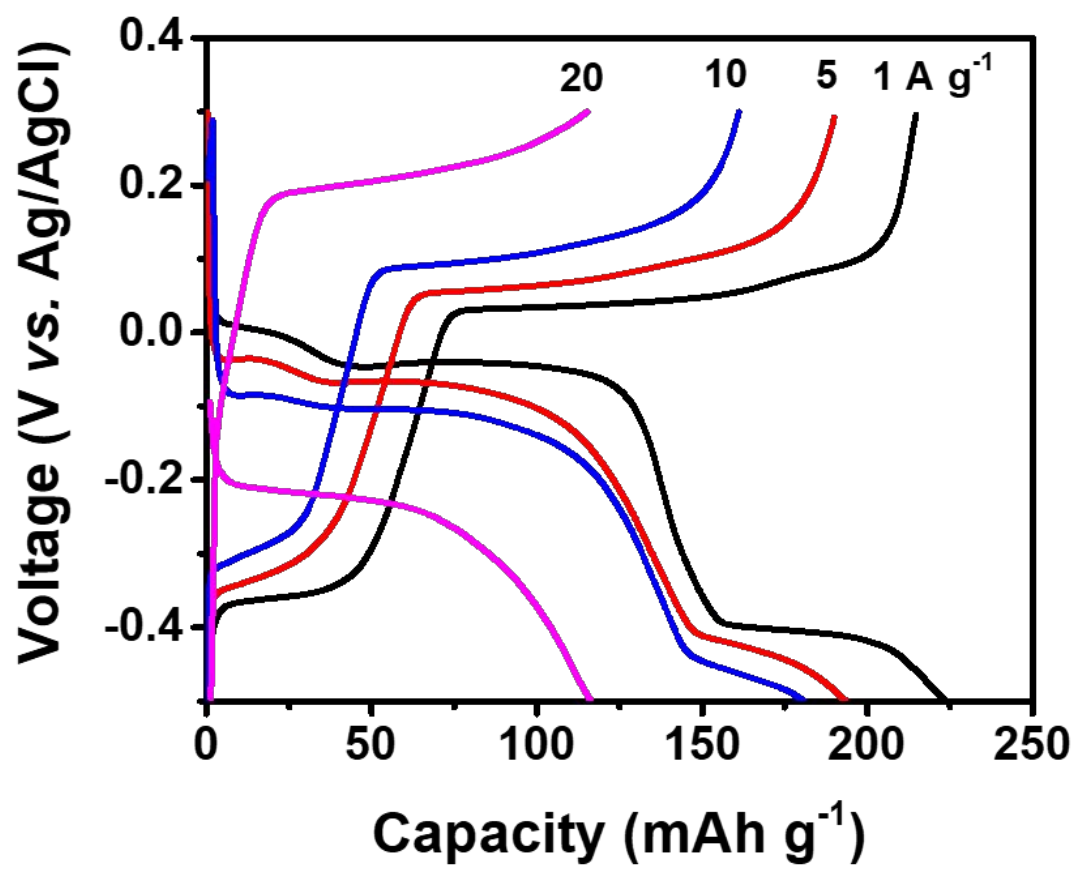

Figure S11 Charge-discharge profiles at the different current of $\mathrm{MoO}_{3} \mathrm{NB}$ electrode. 


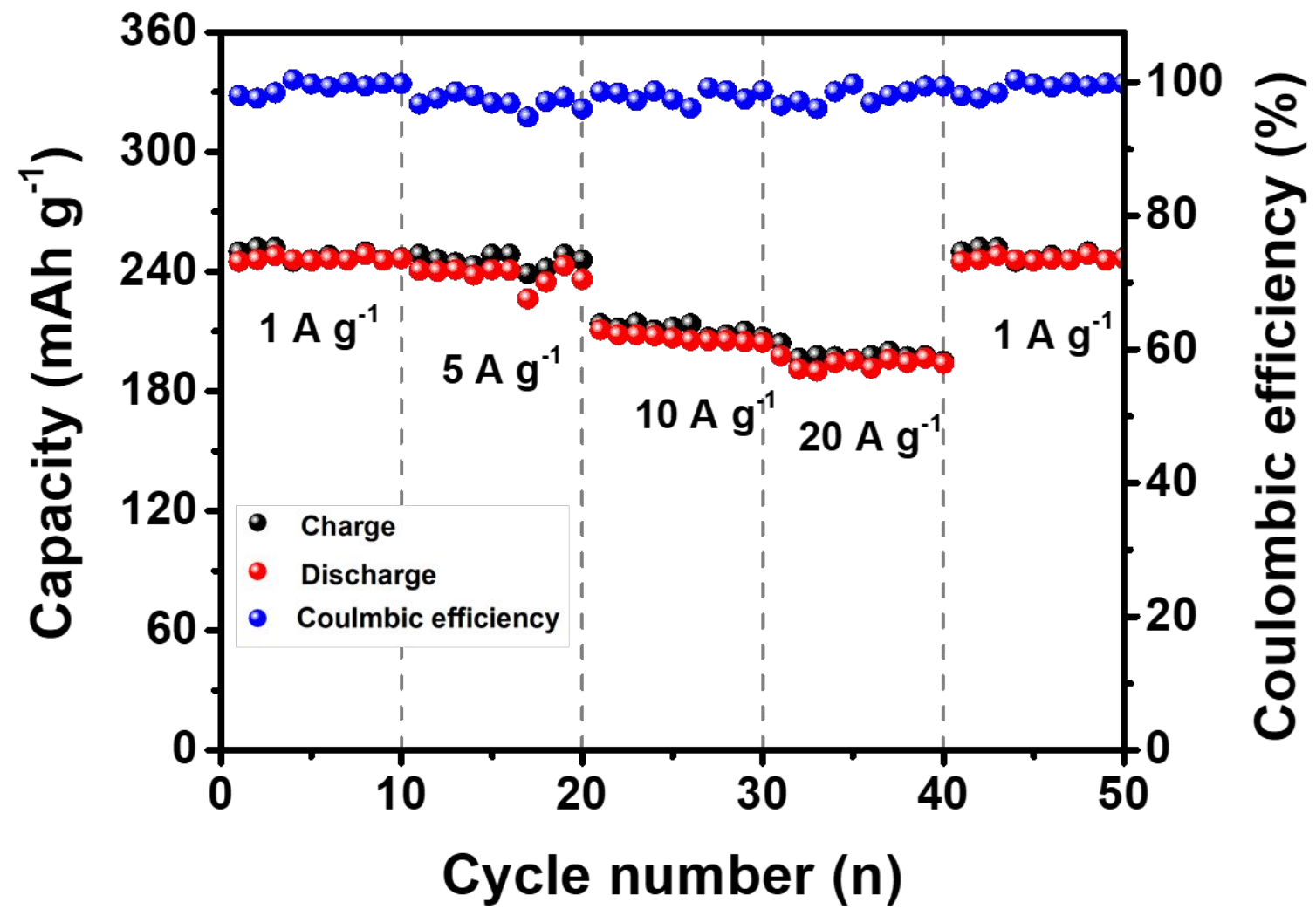

Figure S12 Rate performance at current densities from 1 to $20 \mathrm{~A} \mathrm{~g}^{-1}$ of d-MoO $\mathrm{MNB}_{3}$ electrode. 


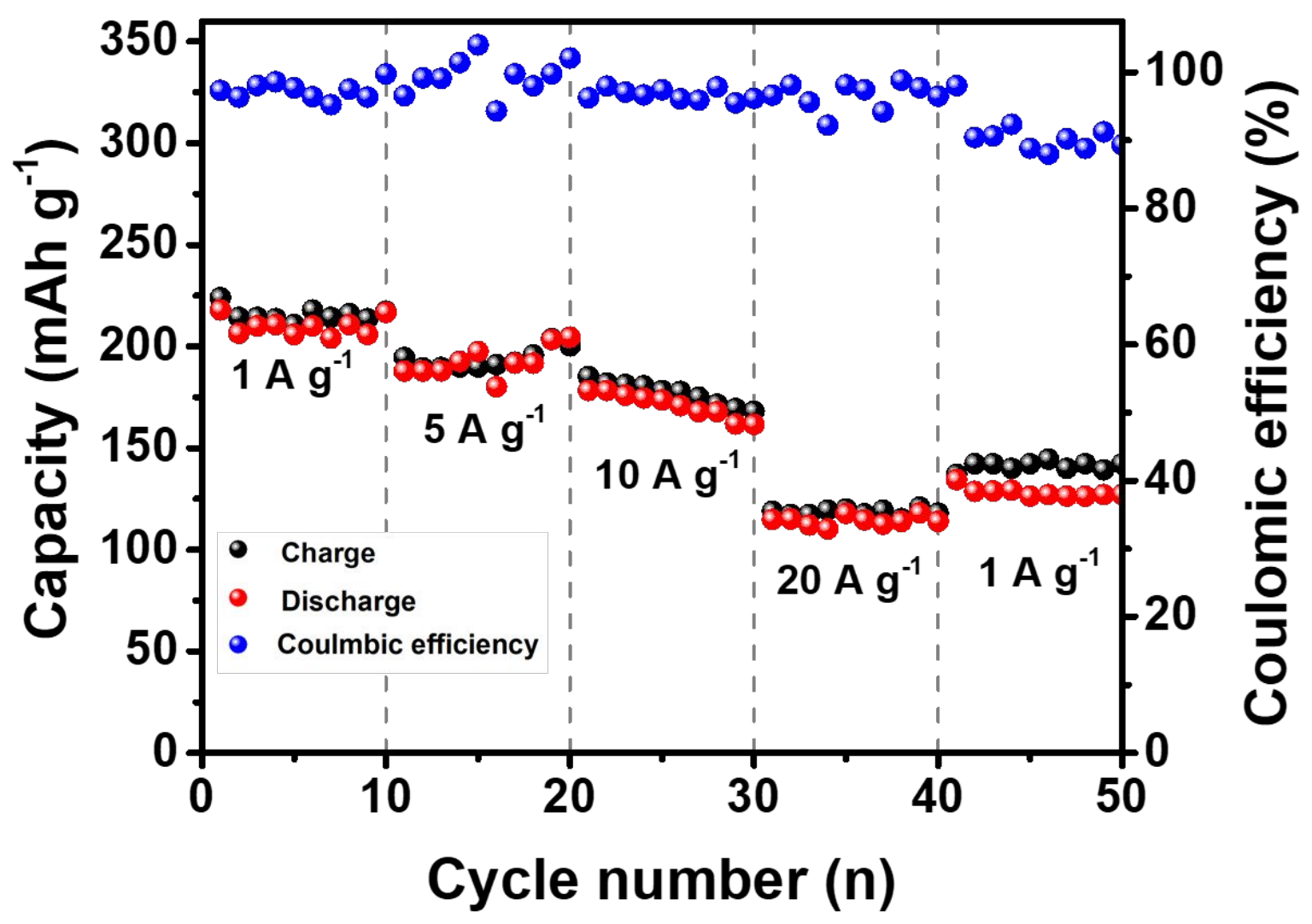

Figure S13 Rate performance at current densities from 1 to $20 \mathrm{~A} \mathrm{~g}^{-1}$ of $\mathrm{MoO}_{3} \mathrm{NB}$ electrode. 


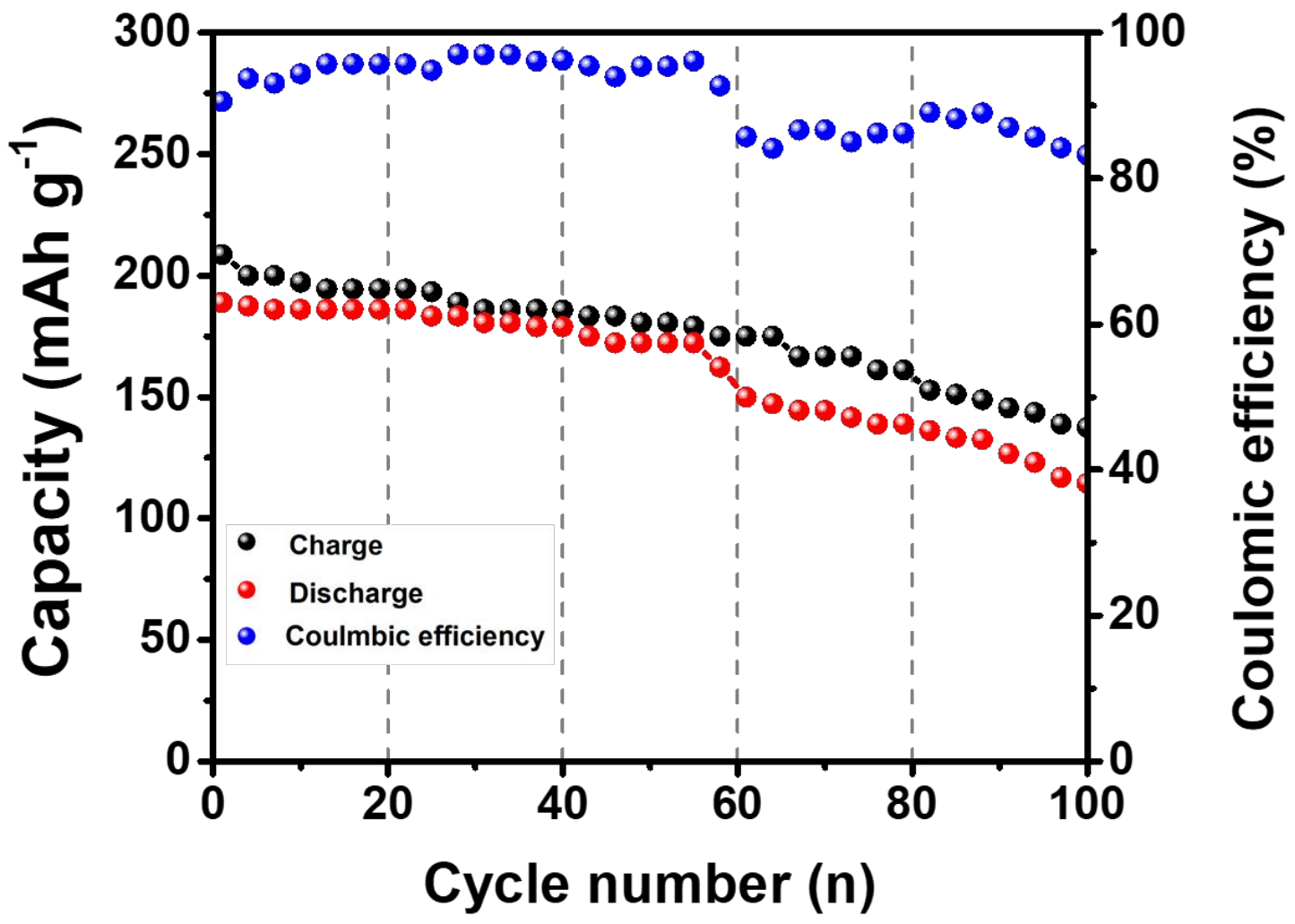

Figure S14 Cycle performance and Coulombic efficiency at a current density of $10 \mathrm{~A} \mathrm{~g}^{-1}$ of $\mathrm{MoO}_{3} \mathrm{NB}$ electrode. 


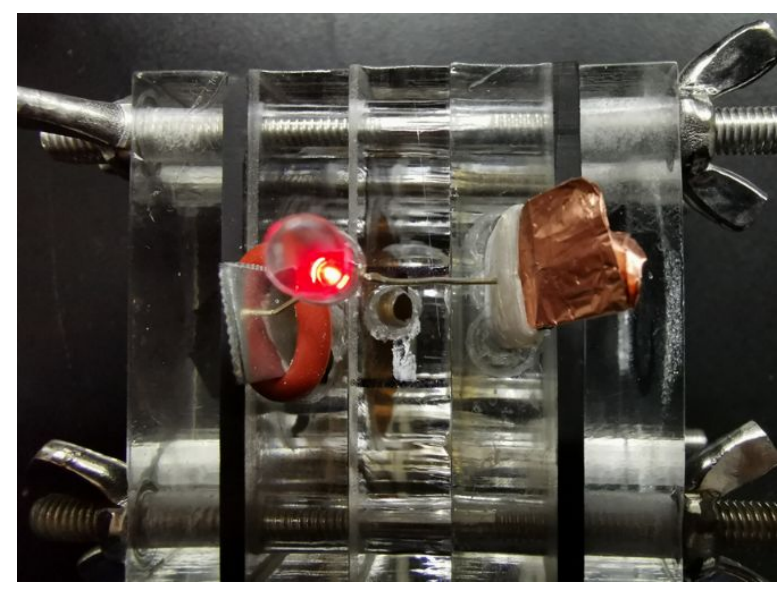

Figure S15 Digital photograph of an alkali-acid $\mathrm{Zn}-\mathrm{MoO}_{3}$ hybrid battery that lighting a red LED. 


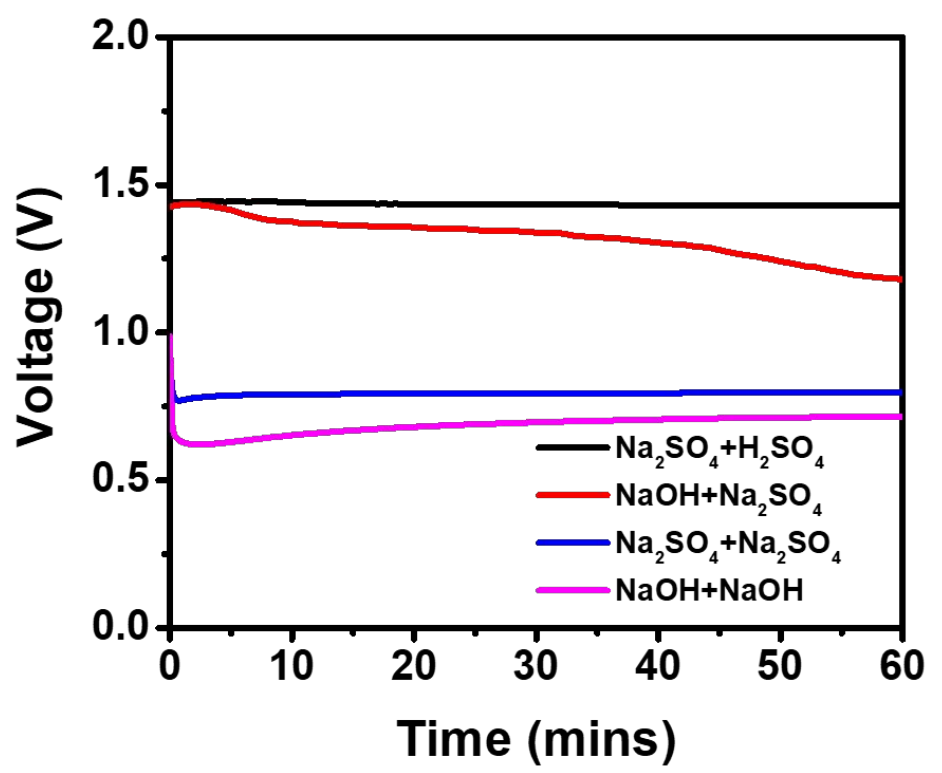

Figure S16 OCV of $\mathrm{Zn}-\mathrm{MoO}_{3}$ battery with various electrolytes. Black line, $1 \mathrm{M} \mathrm{Na}_{2} \mathrm{SO}_{4}$ serve as anolyte and $2 \mathrm{M} \mathrm{H}_{2} \mathrm{SO}_{4}$ serve as catholyte; red line, $4 \mathrm{M} \mathrm{NaOH}$ serve as anolyte and $1 \mathrm{M}$ $\mathrm{Na}_{2} \mathrm{SO}_{4}$ serve as catholyte; Blue line, $1 \mathrm{M} \mathrm{Na}_{2} \mathrm{SO}_{4}$ serve as both anolyte and catholyte; Pink line, $4 \mathrm{M} \mathrm{NaOH}$ serve as anolyte and catholyte. 


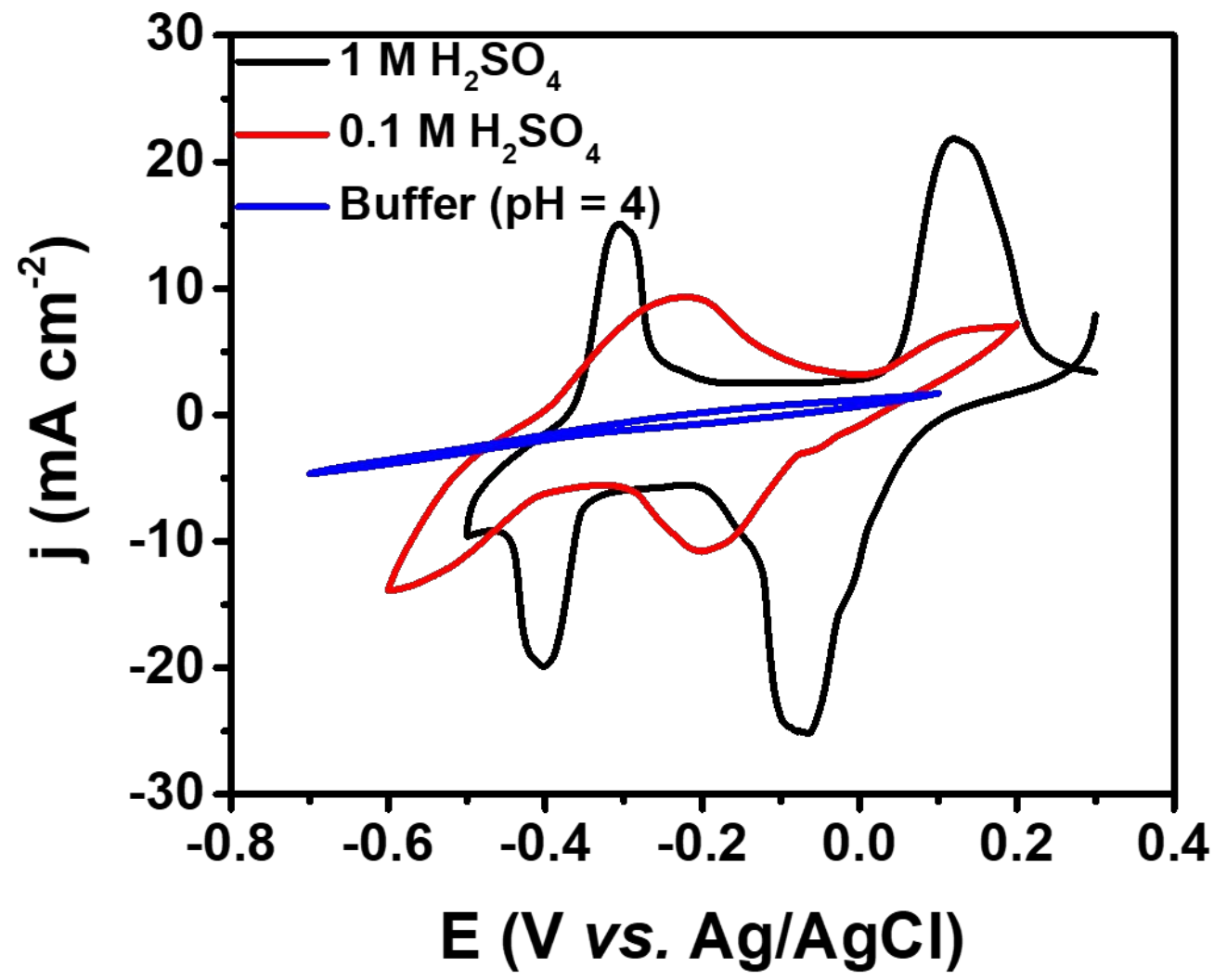

Figure $\mathrm{S17} \mathrm{CV}$ curves of d-MoO${ }_{3} \mathrm{PNB}$ with a scan rate of $50 \mathrm{mV} \mathrm{s}^{-1}$ in different conditions. 


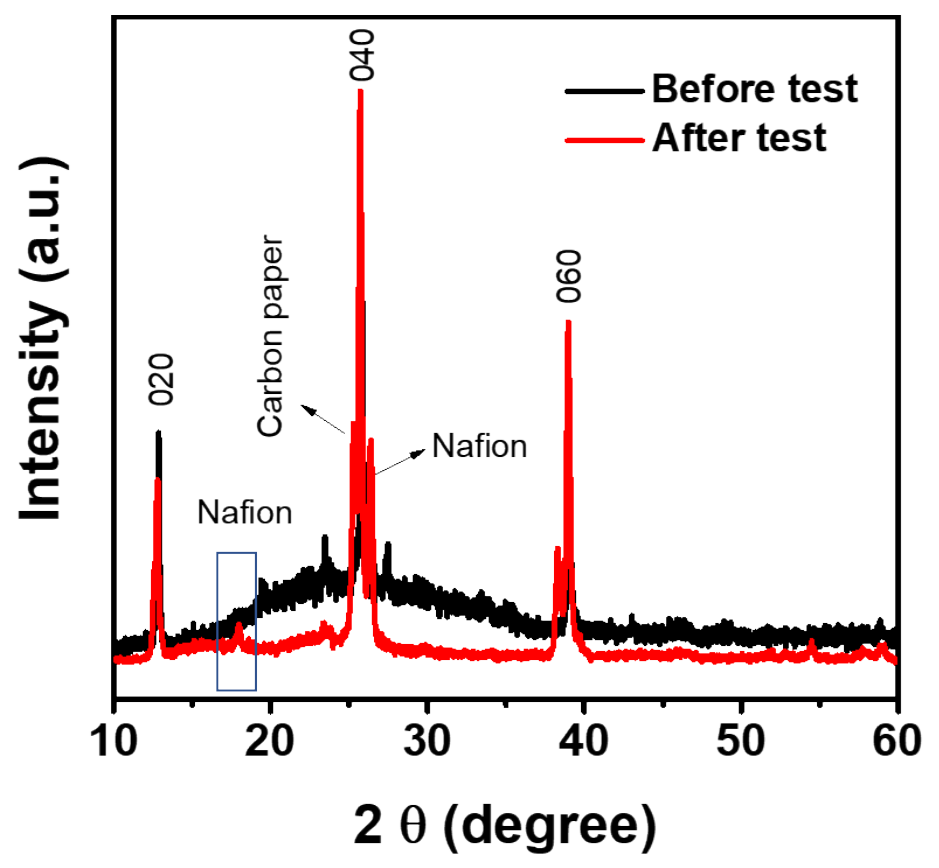

Figure S18 XRD pattern of d- $\mathrm{MoO}_{3}$ PNB before and after long-term stability test. 

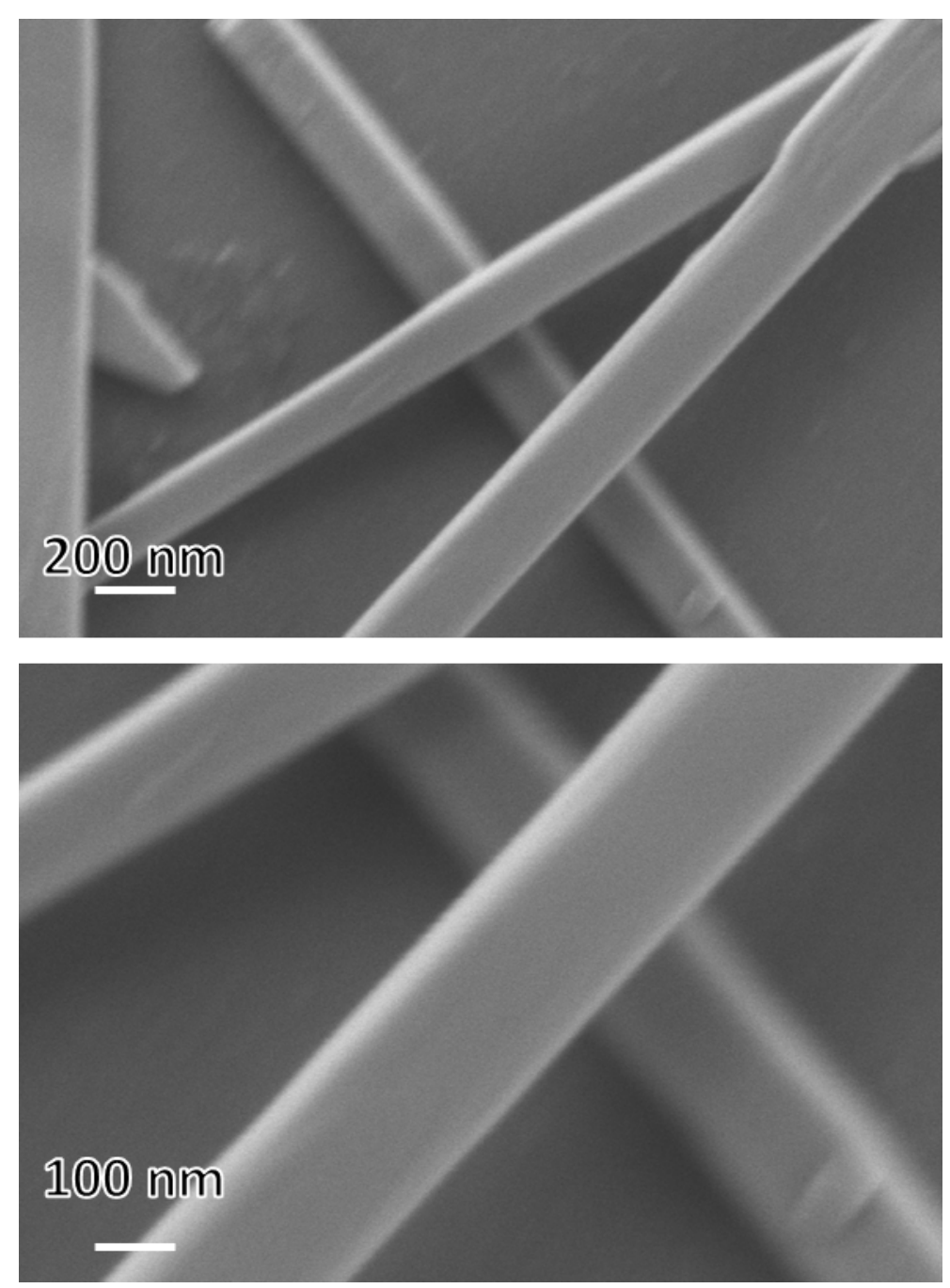

Figure S19 SEM images of d- $\mathrm{MoO}_{3}$ PNB after long-term stability test. 

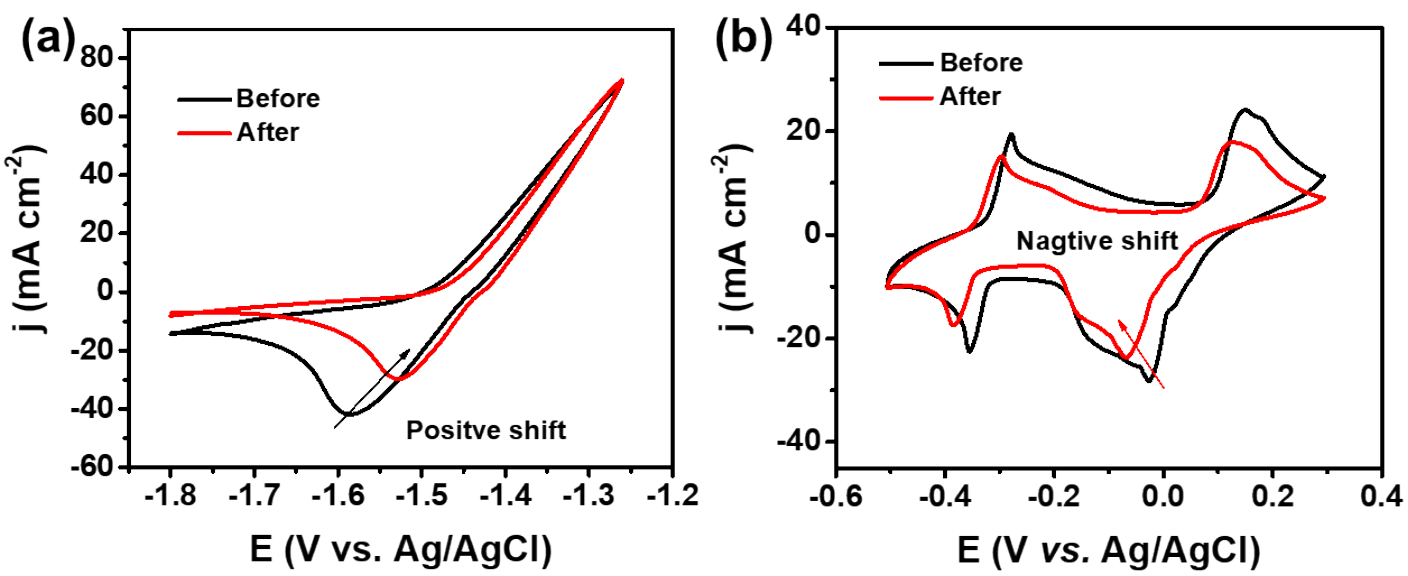

Figure S20 (a) CV curves of Zn anode in the anolyte before and after stability test with a scan rate of $10 \mathrm{mV} \mathrm{s}^{-1}$. (b) $\mathrm{CV}$ curves of $\mathrm{d}-\mathrm{MoO}_{3} \mathrm{PNB}$ in the catholyte before and after stability test with a scan rate of $50 \mathrm{mV} \mathrm{s}^{-1}$. 


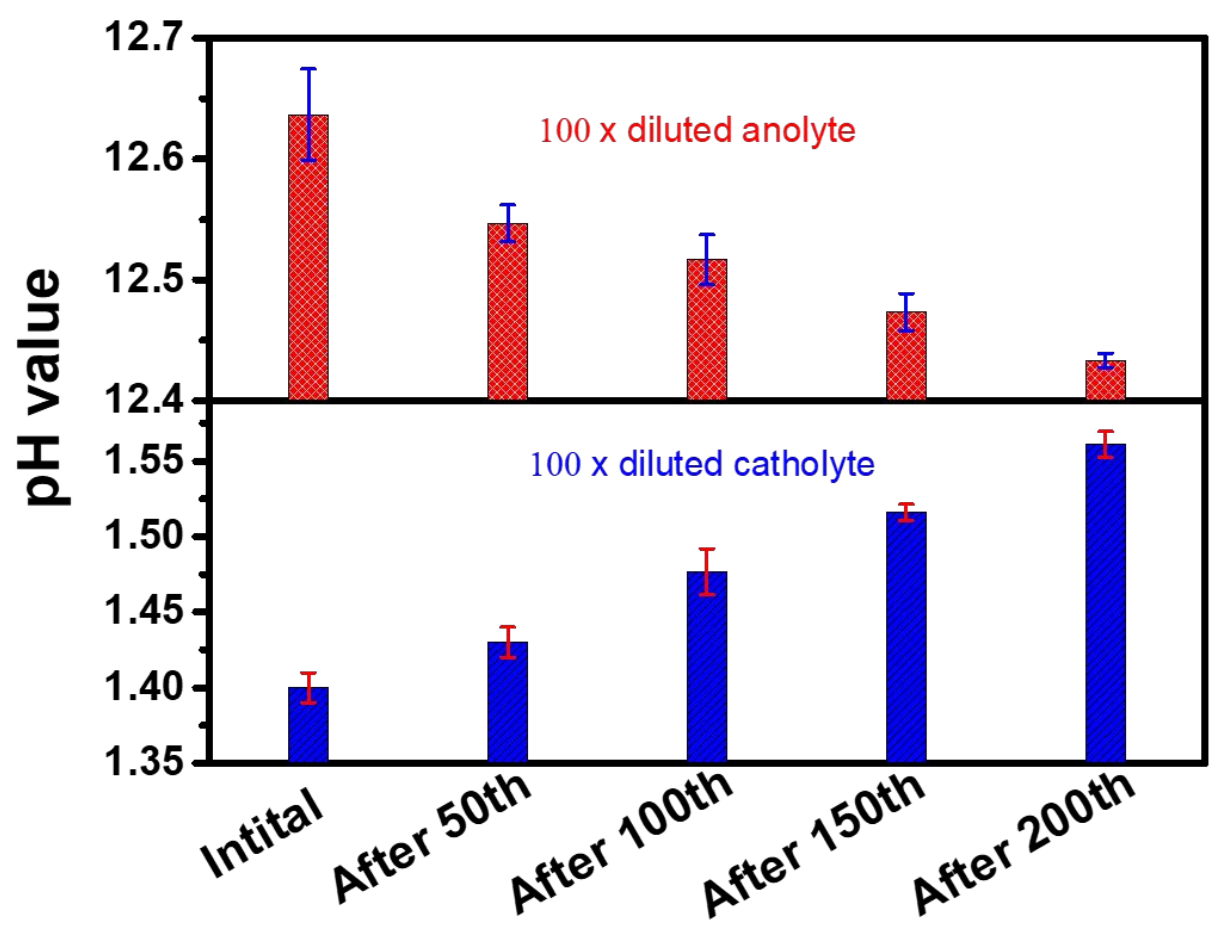

Figure S21 The $\mathrm{pH}$ variation of anolyte and catholyte during the charge-discharge process alkali-acid $\mathrm{Zn}-\mathrm{MoO}_{3}$ hybrid battery. 


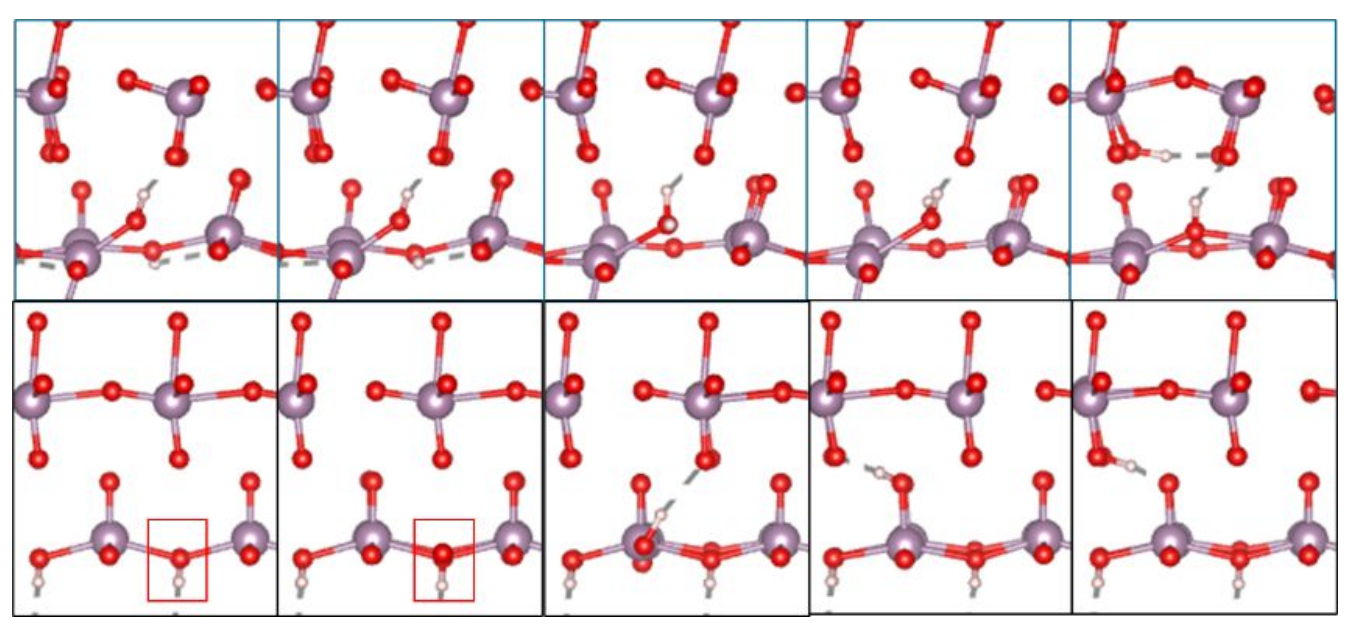

Figure S22 The snapshots for intact and defective Dv and Dp2. 
Table S1 The performance comparison of various types of aqueous batteries.

\begin{tabular}{|c|c|c|c|c|c|c|c|}
\hline & Battery type & $\begin{array}{l}\text { Voltage } \\
\text { (V) }\end{array}$ & $\begin{array}{l}\text { Capacity } \\
\left(\mathrm{mAh} \mathrm{g}^{-1}\right. \\
\left.\text { at } 1 \mathrm{~A} \mathrm{~g}^{-1}\right)\end{array}$ & 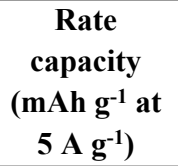 & $\begin{array}{c}\text { Energy } \\
\text { density } \\
\left(\mathrm{Wh} \mathrm{Kg}^{-1}\right)\end{array}$ & $\begin{array}{l}\text { Cycle } \\
\text { stability }\end{array}$ & Reference \\
\hline \multirow{4}{*}{$\begin{array}{l}\text { Dual-ion } \\
\text { battery }\end{array}$} & $\mathrm{Zn} / / \mathrm{Na}_{0.44} \mathrm{MnO}_{2}$ & 1.5 & $<50$ & $<40$ & 1 & $\begin{array}{c}73 \% \text { after } \\
1000 \text { cycles }\end{array}$ & 1 \\
\hline & $\mathrm{Al} / / 3 \mathrm{DGF}$ & 2.2 & 132 & $<100$ & / & $\begin{array}{l}75 \% \text { after } \\
150 \text { cycles }\end{array}$ & 2 \\
\hline & $\mathrm{Cu}_{3}\left(\mathrm{PO}_{4}\right)_{2} / / \mathrm{Na}_{0.44} \mathrm{MnO}_{2}$ & 1.1 & $\begin{array}{c}52.6 \text { at } 0.5 \\
\text { C }\end{array}$ & I & l & $\begin{array}{l}60 \% \text { after } \\
30 \text { cycles }\end{array}$ & 3 \\
\hline & $\mathrm{Mn}_{3} \mathrm{O}_{4}$ & l & 52 & 30 & l & $\begin{array}{l}33 \% \text { after } \\
3500 \text { cycles }\end{array}$ & 4 \\
\hline \multirow{5}{*}{$\begin{array}{l}\text { Zn-ion } \\
\text { battery }\end{array}$} & $\mathrm{H}_{2} \mathrm{~V}_{3} \mathrm{O}_{8}$ & 1.6 & 240.5 & 113.9 & 1 & $\begin{array}{l}94 \% \text { after } \\
1000 \text { cycles }\end{array}$ & 5 \\
\hline & $\mathrm{Na}_{2} \mathrm{~V}_{2}(\mathrm{PO} 4)_{2} \mathrm{~F}_{3}$ & 1.9 & 50 & $<30$ & 97.5 & $\begin{array}{l}95 \% \text { after } \\
4000 \text { cycles }\end{array}$ & 6 \\
\hline & $\mathrm{MnO}_{2} / \mathrm{GCF}$ & 1.8 & 258.5 & $<94.8$ & 123.5 & $\begin{array}{l}73 \% \text { after } \\
1000 \text { cycles }\end{array}$ & 7 \\
\hline & $\mathrm{Cu}_{2-\mathrm{x}} \mathrm{Se}$ & 0.9 & 83.8 & 40.1 & 69.6 & $\begin{array}{l}84 \% \text { after } \\
120 \text { cycles }\end{array}$ & 8 \\
\hline & $\mathrm{MoS}_{2}-\mathrm{O}$ & 1.4 & 98 & l & $<50$ & $\begin{array}{l}61 \% \text { after } \\
1000 \text { cycles }\end{array}$ & 9 \\
\hline \multirow{3}{*}{$\begin{array}{l}\mathrm{H}^{+} \text {ion } \\
\text { battery }\end{array}$} & Mxene//Quinone & 1.6 & $<80$ & 1 & $<100$ & $\begin{array}{l}90 \% \text { after } \\
5000 \text { cycles }\end{array}$ & 10 \\
\hline & $\mathrm{MoO}_{3} / / \mathrm{MnO}_{2}$ & 1.5 & 209 & l & 177.4 & $\begin{array}{l}81 \% \text { after } \\
300 \text { cycles }\end{array}$ & 11 \\
\hline & $\mathrm{MoO}_{3}$ & / & 152 & 123 & 1 & $\begin{array}{l}67 \% \text { after } \\
100 \text { cycles }\end{array}$ & 12 \\
\hline \multirow{2}{*}{$\begin{array}{l}\mathrm{NH}_{4}{ }^{+} \text {ion } \\
\text { battery }\end{array}$} & $\begin{array}{c}\left(\mathrm{NH}_{4}\right)_{1.47} \mathrm{Ni}\left[\mathrm{Fe}(\mathrm{CN})_{6}\right]_{0.88} / / \\
\text { PTCDI }\end{array}$ & 1.7 & 23 & 1 & 43 & $\begin{array}{l}67 \% \text { after } \\
1000 \text { cycles }\end{array}$ & 13 \\
\hline & $\begin{array}{c}\mathrm{Fe}\left[\mathrm{Fe}(\mathrm{CN})_{6}\right] / / \\
\left(\mathrm{NH}_{4}\right) \mathrm{Fe}\left[\mathrm{Fe}(\mathrm{CN})_{6}\right]\end{array}$ & 1.1 & 50 & l & 38 & $\begin{array}{l}53 \% \text { after } \\
600 \text { cycles } \\
\end{array}$ & 14 \\
\hline \multicolumn{2}{|r|}{$\mathrm{Zn}-\mathrm{MoO}_{3}$ battery } & 1.85 & 198.2 & 157.8 & 273 & $\begin{array}{l}>90 \% \text { after } \\
200 \text { cycles }\end{array}$ & This work \\
\hline
\end{tabular}




\section{Reference}

1. Yuan, T.; Zhang, J.; Pu, X.; Chen, Z.; Tang, C.; Zhang, X.; Ai, X.; Huang, Y.; Yang, H.; Cao, Y., Novel Alkaline Zn/Na0.44MnO2 Dual-Ion Battery with a High Capacity and Long Cycle Lifespan. ACS Appl. Mater. Interfaces 2018, 10 (40), 34108-34115, DOI 10.1021/acsami.8b08297.

2. Zhang, E.; Cao, W.; Wang, B.; Yu, X.; Wang, L.; Xu, Z.; Lu, B., A novel aluminum dual-ion battery. Energy Storage Materials 2018, 11, 91-99, DOI 10.1016/j.ensm.2017.10.001.

3. Yu, H.; Deng, C.; Yan, H.; Xia, M.; Zhang, X.; Wang, Z.-B.; Shu, J., $\mathrm{Cu}_{3}\left(\mathrm{PO}_{4}\right)_{2}$ : Novel Anion Convertor for Aqueous Dual-Ion Battery. Nano-Micro Letters 2021, 13 (1), 1-8, DOI 10.1007/s40820-020-00576-1.

4. Jiang, H.; Wei, Z.; Ma, L.; Yuan, Y.; Hong, J. J.; Wu, X.; Leonard, D. P.; Holoubek, J.; Razink, J. J.; Stickle, W. F.; Du, F.; Wu, T.; Lu, J.; Ji, X., An Aqueous DualIon Battery Cathode of $\mathrm{Mn}_{3} \mathrm{O}_{4}$ via Reversible Insertion of Nitrate. Angew. Chem. Int. Ed. Engl 2019, 58 (16), 5286-5291, DOI 10.1002/anie.201814646.

5. He, P.; Quan, Y.; Xu, X.; Yan, M.; Yang, W.; An, Q.; He, L.; Mai, L., HighPerformance Aqueous Zinc-Ion Battery Based on Layered $\mathrm{H}_{2} \mathrm{~V}_{3} \mathrm{O}_{8}$ Nanowire Cathode. Small 2017, 13 (47), 1702551, DOI 10.1002/smll.201702551.

6. Li, W.; Wang, K.; Cheng, S.; Jiang, K., A long-life aqueous Zn-ion battery based on $\mathrm{Na}_{3} \mathrm{~V}_{2}\left(\mathrm{PO}_{4}\right) 2 \mathrm{~F}_{3}$ cathode. Energy Storage Materials 2018, 15, 14-21, DOI 10.1016/j.ensm.2018.03.003.

7. Wu, Y.; Wang, M.; Tao, Y.; Zhang, K.; Cai, M.; Ding, Y.; Liu, X.; Hayat, T.; Alsaedi, A.; Dai, S., Electrochemically Derived Graphene-Like Carbon Film as a Superb Substrate for High-Performance Aqueous Zn-Ion Batteries. Adv. Funct. Mater. 2020, 30 (5), 1907120, DOI 10.1002/adfm.201907120.

8. Yang, Y.; Xiao, J.; Cai, J.; Wang, G.; Du, W.; Zhang, Y.; Lu, X.; Li, C. C., MixedValence Copper Selenide as an Anode for Ultralong Lifespan Rocking-Chair Zn - Ion Batteries: An Insight into its Intercalation/Extraction Kinetics and Charge Storage Mechanism. Adv. Funct. Mater. 2020, 2005092, DOI 10.1002/adfm.202005092.

9. Liang, H.; Cao, Z.; Ming, F.; Zhang, W.; Anjum, D. H.; Cui, Y.; Cava.llo, L.; Alshareef, H. N., Aqueous zinc-ion storage in MoS2 by tuning the intercalation energy. Nano letters 2019, 19 (5), 3199-3206, DOI 10.1021/acs.nanolett.9b00697. 
10. Hu, M.; Cui, C.; Shi, C.; Wu, Z.-S.; Yang, J.; Cheng, R.; Guang, T.; Wang, H.; Lu, H.; Wang, X., High-Energy-Density Hydrogen-Ion-Rocking-Chair Hybrid Supercapacitors Based on $\mathrm{Ti}_{3} \mathrm{C}_{2} \mathrm{~T}_{\mathrm{x}}$ MXene and Carbon Nanotubes Mediated by Redox Active Molecule. ACS Nano 2019, 13 (6), 6899-6905, DOI 10.1021/acsnano.9b01762.

11. Yan, L.; Huang, J.; Guo, Z.; Dong, X.; Wang, Z.; Wang, Y., Solid-State Proton Battery Operated at Ultralow Temperature. ACS Energy Letters 2020, 5 (2), 685-691, DOI 10.1021/acsenergylett.0c00109.

12. Wang, X.; Xie, Y.; Tang, K.; Wang, C.; Yan, C., Redox Chemistry of Molybdenum Trioxide for Ultrafast Hydrogen-Ion Storage. Angew. Chem. Int. Ed. 2018, 57 (36), 1156911573, DOI 10.1002/anie.201803664.

13. Wu, X.; Qi, Y.; Hong, J. J.; Li, Z.; Hernandez, A. S.; Ji, X., Rocking-Chair Ammonium-Ion Battery: A Highly Reversible Aqueous Energy Storage System. Angew. Chem. Int. Ed. 2017, 56 (42), 13026-13030, DOI 10.1002/anie.201803664.

14. Wu, X.; Xu, Y.; Jiang, H.; Wei, Z.; Hong, J. J.; Hernandez, A. S.; Du, F.; Ji, X., $\mathrm{NH}^{+}$topotactic insertion in Berlin green: an exceptionally long-cycling cathode in aqueous ammonium-ion batteries. ACS Appl. Energy Mater. 2018, 1 (7), 3077-3083, DOI 10.1021/acsaem.8b00789. 